\title{
Unveiling Planar Defects in Hexagonal Group IV Materials
}

\author{
Elham M. T. Fadaly, $\triangle$ Anna Marzegalli, $\triangle$ Yizhen Ren, Lin Sun, Alain Dijkstra, Diego de Matteis, \\ Emilio Scalise, Andrey Sarikov, Marta De Luca, Riccardo Rurali, Ilaria Zardo, Jos E. M. Haverkort, \\ Silvana Botti, Leo Miglio, Erik P. A. M. Bakkers, and Marcel A. Verheijen*
}

Cite This: Nano Lett. 2021, 21, 3619-3625

Read Online

\section{ACCESS |}

山ll Metrics \& More

国 Article Recommendations

Supporting Information

ABSTRACT: Recently synthesized hexagonal group IV materials are a promising platform to realize efficient light emission that is closely integrated with electronics. A high crystal quality is essential to assess the intrinsic electronic and optical properties of these materials unaffected by structural defects. Here, we identify a previously unknown partial planar defect in materials with a type $I_{3}$ basal stacking fault and investigate its structural and electronic properties. Electron microscopy and atomistic modeling are used to reconstruct and visualize this stacking fault and its terminating
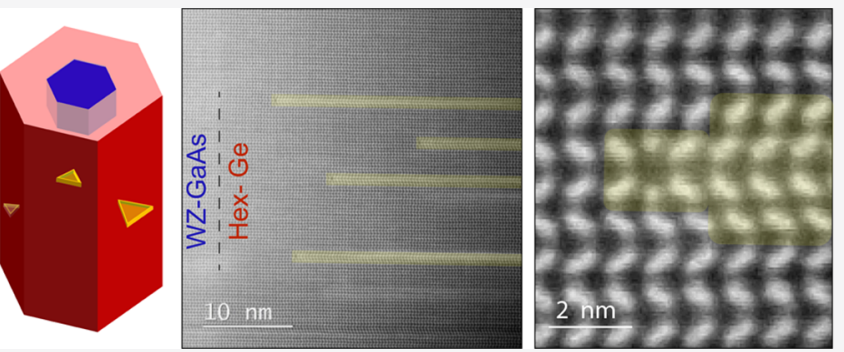
dislocations in the crystal. From band structure calculations coupled to photoluminescence measurements, we conclude that the $I_{3}$ defect does not create states within the hex-Ge and hex-Si band gap. Therefore, the defect is not detrimental to the optoelectronic properties of the hex-SiGe materials family. Finally, highlighting the properties of this defect can be of great interest to the community of hex-III-Ns, where this defect is also present. KEYWORDS: Nanowires, hexagonal group IV, hexagonal Ge, hexagonal Si, $I_{3}$ basal stacking fault, defects

G roup IV materials, especially silicon $(\mathrm{Si})$ and germanium I $(\mathrm{Ge})$, are considered cornerstone materials in the semiconductor industry. Their unique physical and electronic properties in addition to their well-established technology qualify them for a broad range of applications in the fields of electronics and optoelectronics. However, the fundamental limitation is that bulk crystalline cubic $\mathrm{Si}$ and $\mathrm{Ge}$ are incapable of efficiently emitting light due to the indirect nature of their band gaps. This has so far restrained them from being fully utilized in active optical circuitry. Recently, Fadaly et al. ${ }^{1}$ experimentally realized direct band gap $\mathrm{Si}_{1-x} \mathrm{Ge}_{x}$ alloys by exploiting the metastable hexagonal crystal structure. This transformative result can open new frontiers toward uniting the electronic and optoelectronic functionalities on a single chip, paving the way toward the long-held goal of integrated group IV photonics. For this to happen, it is pivotal to master the structural, electronic, and optical properties of this new class of hexagonal group IV materials (i.e., Si and $\mathrm{Ge}$ ), which are mostly unexplored. A high crystalline quality is required to fully understand the intrinsic electronic and optoelectronic properties of the materials, since structural defects can be detrimental to the material properties.

In group IV materials, the hexagonal crystal phase is a metastable phase, which is not readily achievable via conventional thin-film growth techniques and can only be realized under extreme conditions in small volumes, as demonstrated in the literature. ${ }^{2-8}$ Here, a unique feature of bottom-up-grown nanowires is employed-their intrinsic capability to create metastable crystal phases inaccessible by conventional material synthesis techniques in the planar geometry. Capitalizing on this unique attribute, the hexagonal crystal phase has been achieved in group IV materials via the "crystal transfer" technique reported by some of us. ${ }^{1,9-11}$ The hexagonal crystal structure of group IV materials was adopted from a hexagonal nanowire core material template. This technique allows the fabrication of single-crystalline materials in large volumes. ${ }^{1}$

Hexagonal (hex) Ge and Si crystals have been epitaxially grown around almost-lattice-matched wurtzite (WZ) gallium arsenide $(\mathrm{GaAs})$ and gallium phosphide $(\mathrm{GaP})$ nanowires. In this paper, we focus on studying the GaAs/Ge system in detail. The $\mathrm{GaP} / \mathrm{Si}$ system, which displays a similar defect geometry, is discussed in the Supporting Information. The material systems studied in this work were fabricated using metalorganic vapor phase epitaxy (MOVPE) with trimethylgallium (TMGa), arsine $\left(\mathrm{AsH}_{3}\right)$, germane $\left(\mathrm{GeH}_{4}\right)$, phosphine $\left(\mathrm{PH}_{3}\right)$, and tetrasilane $\left(\mathrm{Si}_{4} \mathrm{H}_{10}\right)$ precursors; for the growth details, see the Methods section in the Supporting Information. The growth of $\mathrm{GaAs} / \mathrm{Ge}$ (and $\mathrm{GaP} / \mathrm{Si}$ ) was performed in three main steps. First, gold ( $\mathrm{Au}$ )-catalyzed untapered WZ III-V

Received: February 17, 2021

Revised: April 1, 2021

Published: April 12, 2021 
nanowire arrays were grown with the vapor-liquid-solid (VLS) method. Then, the Au catalysts were chemically removed from the nanowire cores, and hex-Ge and hex-Si shells were eventually epitaxially grown around the Au-free nanowire cores. ${ }^{1}$ A representative scanning electron microscopy (SEM) image in Figure la shows an array of nanowires
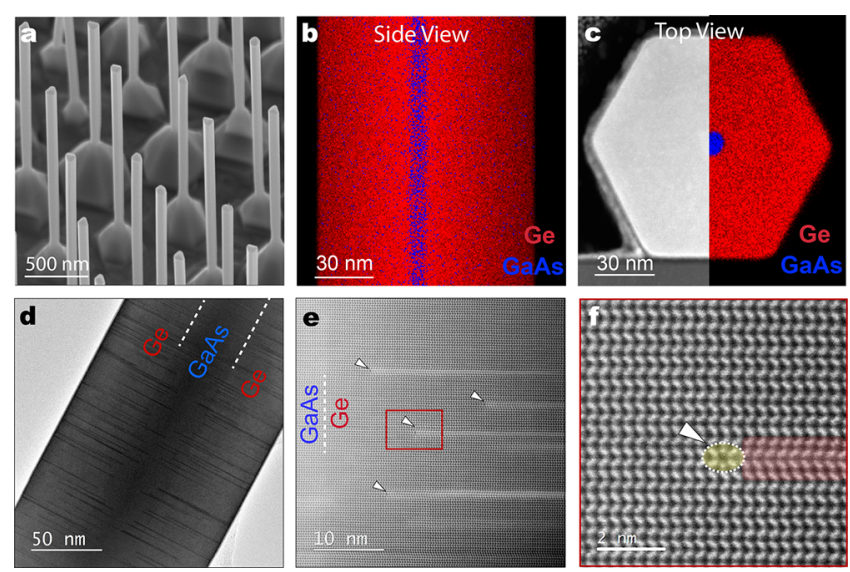

Figure 1. Epitaxial hexagonal GaAs/Ge core/shell nanowires. (a) A representative SEM image of an array of hex-GaAs/Ge core/shell nanowires grown in the $\langle 0001\rangle$ growth direction on a GaAs(111)B substrate. (b) EDX compositional map for the axial direction of one representative $\mathrm{GaAs} / \mathrm{Ge}$ core/shell nanowire. (c) Overlapped HAADF-STEM and EDX images of a cross-sectional thin lamella of a similar nanowire. (d) BF-TEM image of a GaAs/Ge core/shell structure, with white dashed lines as a guide for the eye indicating the interfaces between the two material systems. The dark partial lines across the structure represent the stacking faults in the Ge shell. (e) Aberration-corrected HAADF-STEM zoomed-in image for a region of the GaAs/Ge structure obtained in the $\langle 11 \overline{2} 0\rangle$ zone axis, which indicates the starting position of the partial stacking faults. (f) A magnified image of the framed region in panel e that displays the $A B A B$ stacking of the hexagonal crystal structure of the Ge shell. The red highlighted region designates the double-layer partial-stacking fault, which leads to a $4 \mathrm{H}$-polytype-like stacking that originates from the terminating partial dislocations encircled in yellow and indicated by a white arrow.

with thin hex-Ge shells around thin WZ-GaAs cores. The elemental composition of the nanowires was studied by energy-dispersive X-ray spectroscopy (EDX) using transmission electron microscopy (TEM). The elemental maps across the axial and radial directions of the wires, as depicted in Figure $\mathrm{lb}$ and $\mathrm{c}$, respectively, confirm that the GaAs/Ge core/ shell structure shows no intermixing between GaAs and Ge. A detailed analysis of the crystal structure of hex-Ge reveals the presence of one predominant type of defect. This type of defect appears in bright field (BF) TEM images as dark lines, starting from random spots in the hexagonal shell and continuing toward the edge of the shell as presented in Figure 1d and, for hex-Si, Supporting Figure, 1a. In the high-angle annular dark-field scanning TEM (HAADF-STEM) images, bright lines are visible, indicating the contribution of the diffraction contrast to the atomic number contrast (see Figure 1e and Supporting Figure 1b). The formation of the defect is intriguing for two main reasons: (1) there is no significant lattice strain present between the core and the shell of our nanowires to be released in the form of a defect and (2) the defects are absent in the core nanowires, as elucidated in Supporting Figure 2, and nucleate in the shell, and their formation seems to be intrinsic to the hexagonal group IV system. The atomic arrangement of hex-Ge and the defect, in particular, was studied in the high-resolution STEM images shown in Figure 1f and Supporting Figure 1c. The defect was revealed to be composed of two stacking fault planes and the related terminating dislocations. In the defect-free part of the hex-Ge crystal, the atomic layers were found to follow perfect $-\mathrm{ABAB}$ - stacking, verifying the hexagonal phase of the material. The stacking-fault terminating dislocations are highlighted by a yellow circle, and the altered stacking sequence is highlighted in red. It is worth pointing out that there is exactly the same number of atomic planes on either side of the terminating dislocations. The change in the dumbbell orientation occurs at the dislocation cores (see Figure 1f). The stacking fault extends to the edge of the core/ shell structure, as clarified in detail in Supporting Figure 3. The result is a planar structure consisting of four-layer stacking that resembles the $4 H$-polytype stacking sequence. ${ }^{12}$ The altered stacking sequence is -ABCBA-, which is identical to the $I_{3}$ basal stacking fault (BSF) type. ${ }^{13}$ The $I_{3}$ BSF was theoretically predicted in 1998 in WZ III-V semiconductors such as AlN, $\mathrm{GaN}$, and InN. ${ }^{14}$ It was first observed experimentally in WZ$\mathrm{GaN}$ in only 2005, showing the faulty stacked planes when viewed from the $\mathrm{WZ}\langle 1 \overrightarrow{120}\rangle$ zone axis. ${ }^{13}$ Neither of these studies considered the possibility of the stacking fault termination being in the bulk of the material instead of at the surface.

To fully characterize the in-plane boundaries of the defect, an extensive BF-TEM study was performed on a thin GaAs/Ge core/shell nanowire. The same section of the core/shell structure was imaged along two different $\langle 11 \overline{2} 0\rangle$ zone axes orthogonal to the $\langle 0001\rangle$ long axis of the nanowire, with a mutual difference in viewing angle of $60^{\circ}$. The $\langle 11 \overline{2} 0\rangle$ zone axis is equivalent to the cubic $\langle 110\rangle$ axis, and both allow for a discrimination between the cubic and hexagonal stacking sequence and the identification of stacking faults in either of the lattices. An ensemble of partial dislocations is visible in the BF-TEM images, as shown in Figure $2 a$ and $b$, appearing as dark lines that extend radially. The positions of the dark lines in the top TEM images are illustrated by colored lines in the bottom panels. Seven defects of different lengths can be clearly distinguished along the two-zone axes. Imaging the defects along the two different zone axes allows the reconstruction of the in-plane shape of the seven defects. In Figure 2c, the defects are projected on the hexagonal cross-sectional core/ shell shape. From this analysis, we can conclude that two dislocation pairs terminate this planar defect with a mutual angle of $60^{\circ}$ and the layers between the dislocations form the $I_{3}$ BSF. It is worth mentioning that BF-TEM imaging is more suited to determine the defect density and the in-plane extension than aberration-corrected HAADF-STEM imaging due to the limited depth-of-focus of the latter despite its better visualization of the atomic arrangement at the dislocations (see Supporting Figure 4 and Supporting Information S1 for more details). ${ }^{15}$ To further confirm that the structure of the dominant defect, which is intrinsic to hexagonal group IV materials, is the $I_{3}$ BSF and not another type of planar defect, we have compared it to similar defects in the literature, as elucidated in Supporting Information S2. ${ }^{16,17}$

Atomistic modeling and $a b$ initio density functional theory (DFT) calculations corroborate the structural characterization inferred by the TEM analysis, as schematically illustrated in Figure 3a. A hexagonally shaped GaAs nanowire (blue) with 


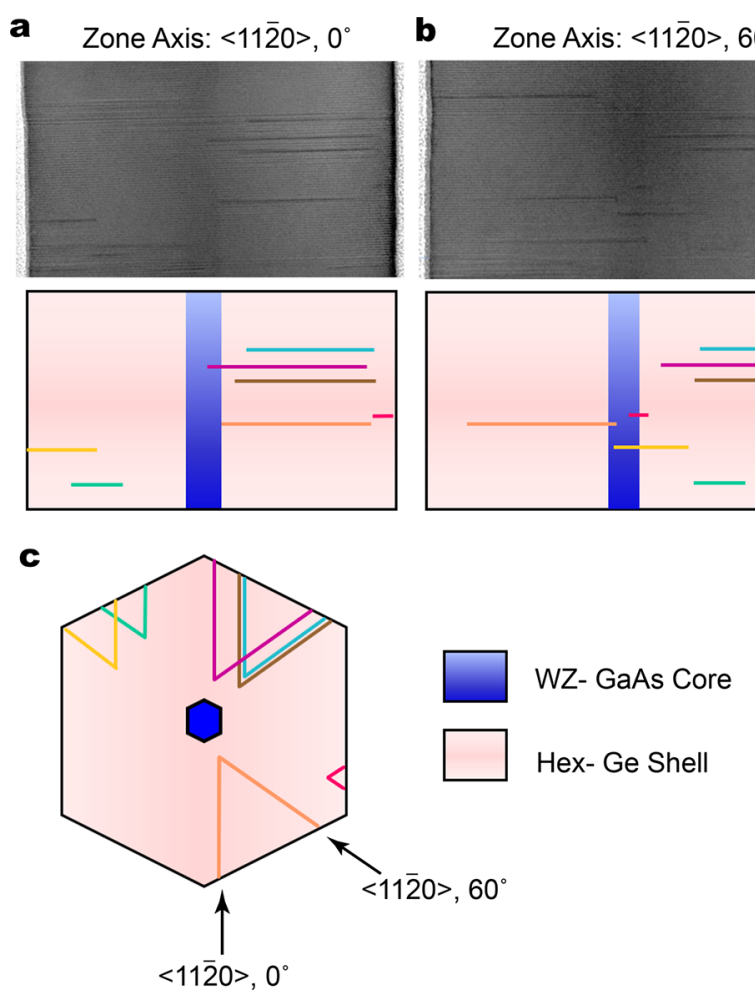

Figure 2. Reconstruction of the in-plane shape of the partial defects. ( $a$ and $b$ ) The top panels show BF-TEM images of the same section of a $9 \mathrm{~nm}$ thick GaAs core and a $45 \mathrm{~nm}$ thick Ge shell nanowire, which were imaged along two $\langle 11 \overline{2} 0\rangle$ zone axes orthogonal to the $\langle 0001\rangle$ long axis of the nanowire. Several partial dislocations are visible. The bottom panels show a schematic illustration of the GaAs/ $\mathrm{Ge}$ core/shell structure, and the colored horizontal lines indicate the positions of seven defects that can clearly be recognized along the two different zone axes. (c) A top view for the reconstruction of the inplane shape of the seven defects indicated in the BF-TEM images, which was created by projecting the defects into a hexagonal shape. All defects have symmetrically equivalent in-plane shapes. All defects have their origin in the shell, i.e., remote from the core/shell interface.

$\{1 \overline{1} 00\}$ facets and a Ge shell (red) with hexagonal crystal lattice positions was constructed. Analytical expressions for the displacement field of the straight dislocations were used to insert two $30^{\circ}$ partial dislocations with opposite Burgers vectors in a basal plane along two $\langle 11 \overline{2} 0\rangle$ directions with an angle of $60^{\circ}$ between them (labeled A in Figure $3 \mathrm{~b}$ ), starting from an arbitrary point. ${ }^{18}$ The same approach was repeated in the subsequent basal plane (labeled B in Figure 3b). In the upper and lower defected planes, opposite Burgers vectors for the dislocation pairs were chosen. Molecular dynamics (MD) simulations using a Tersoff potential were performed, which allowed the group IV atoms to rearrange in their minimum energy configuration (see the Methods section of the Supporting Information for the MD simulations details). ${ }^{19}$ The terminations of the $I_{3}$ BSF, as seen in Figure $1 \mathrm{f}$ and Supporting Figure 1c, are fully reproduced and confirmed as a minimum energy configuration by MD. In Figure 3, atoms belonging to the dislocation cores are colored in green and blue; the same colors indicate the same sign of the Burgers vector. The $I_{3} \mathrm{BSF}$ formation in the internal triangular region is a natural consequence of the insertion of dislocations, which are indeed partial dislocation dipoles on each plane. Figure $3 \mathrm{~b}$ shows that the termination of the $I_{3}$ BSF can be observed only when looking along a $\langle 11 \overline{2} 0\rangle$ viewing direction parallel to its dislocation line, while the dislocations not parallel to the viewing direction cannot be clearly identified, as confirmed by TEM analysis (see Supporting Figure 6).

Notice that we consider the $60^{\circ}$ boundary, terminating the faulty stacked region in each basal plane, as two different dislocations with opposite Burgers vectors. These two dislocations have the same origin and both point toward the nanowire surface, as illustrated in Supporting Figure 7a. However, it should be highlighted that these two terminating dislocations can also be described as a single partial dislocation that forms a semiloop and is characterized by a unique Burgers vector, as seen in Supporting Figure 7b. Mathematically, the latter description means a simultaneous reversal of both the line direction and the Burgers vector of one of the dislocation arms in each basal plane with respect to the first description. Such procedure has no effect on the real atomic arrangement, so both the mentioned descriptions of the boundary of the stacking-fault regions are equivalent.

Although the identification of the nucleation mechanism needs further investigation, the tendency of the system to introduce the illustrated specific defect $\left(I_{3}\right.$ BSFs with pairs of $30^{\circ}$ partial dislocations terminating them) can be explained via energetic considerations. Stacking faults in the hexagonal structure introduce cubically stacked layers in the perfect hexagonal structure. Since the cubic phase is the ground state of bulk $\mathrm{Ge}$ and $\mathrm{Si}$, these stacking faults in hex-Ge and hex-Si have negative formation energies. The energy gain is proportional to the number of cubic rows formed, which is two in this case as shown in Supporting Figure 8c, contrary to the case of a single stacking fault that forms a unique row of cubic sites. Due to the null total Burgers vector, the deformation field induced by the partial dislocations pairs is negligible, and their core energies are predicted to be low. ${ }^{18}$ Moreover, there is no interaction between the two dislocation pairs, which is again due to the null total Burgers vector of each pair, allowing the double-stacking fault to be stable and evolve until the maximum possible extension. The characteristic zero total Burgers vector of the pairs also explains their stability with respect to the nanowire free surface; no net image force is acting on them, so they cannot be expelled. Moreover, it is confirmed that the dislocation nucleation is not due to strain release because null Burgers vector defects cannot release strain, which is consistent with the negligible lattice mismatch between the core and the shell materials.

A multiscale approach was employed to investigate the electronic properties of the $I_{3}$ BSF and its terminating dislocations, considering the defect supercell model shown in Figure $3 a$ and $b$. A smaller atomistic model, as shown in Supporting Figure 9, was used to perform the atomistic relaxation and obtain the minimum energy geometry, as depicted in Figure 3c. Details of the DFT calculations are provided in the Methods section of the Supporting Information. The band structure diagram shows the local density of states (LDOS) along the $\langle 10 \overline{1} 0\rangle$ crystallographic direction, which contains the $I_{3}$ BSF and the dislocations embedded in the hex-Ge crystal averaged in the perpendicular plane (the $<0001>$ direction). ${ }^{20}$ Notably, the LDOS across the $\langle 10 \overline{10}\rangle$ direction in Figure 3c shows no states in the band gap associated with the $I_{3}$ BSF or the dislocation cores. Intuitively, this can be understood by the fact that cubic layers are formed at the stacking fault, which have a larger bandgap than the hexagonal crystal and result in states in the conduction band. Similar results were obtained for hex-Si crystals, as explained in 

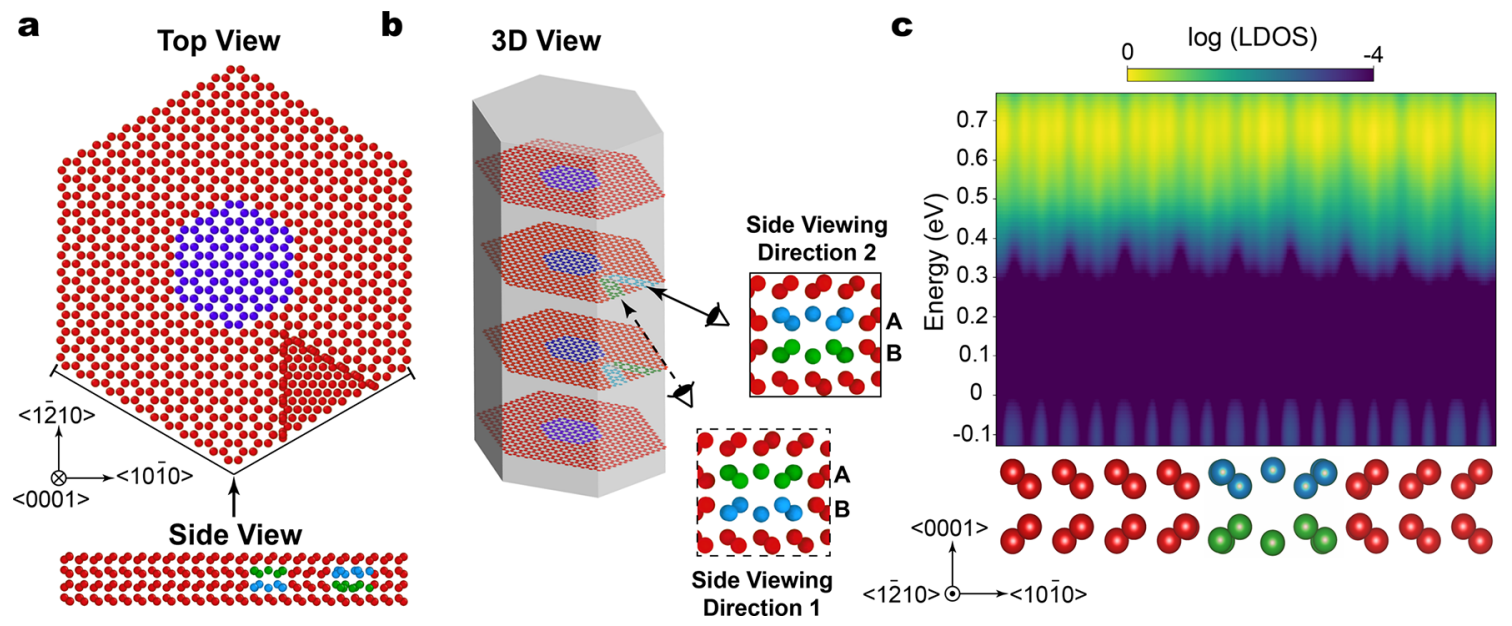

Figure 3. Atomistic model and electronic band structure calculations of $I_{3}$ BSF in hex-Ge and their terminating dislocations. The WZ- GaAs nanowire core atoms are represented in blue, and the hex-Ge shell atoms are represented in red. (a) Top-view atomic model of four atomic layers of the structure containing $I_{3}$ BSF and four bounding partial dislocations. There are two at each stacking fault plane, which are separated with a $60^{\circ}$ angle. The bottom shows a side-view atomic model of the area indicated by the black lines, which is viewed from the direction of the arrow in the top panel. It displays the atomic arrangement at both partial dislocation pairs; for more details on the imaging directions, see Supporting Figure 4. (b) Three-dimensional view for the visualization of the four modeled layers in panel a; the top-most and bottom-most layers are defect-free layers, and the middle layers contain the partial dislocations that enclose the planar stacking faults. A refers to the top defected layer, and B refers to the bottom defected layer. The dislocations, as seen along two different $\langle 11 \overline{2} 0\rangle$ viewing directions, are shown in the framed boxes with dashed and solid lines, which indicate viewing directions 1 and 2, respectively. Blue and green atomic colors highlight the dislocations in the upper and lower defected planes, and the same color means an equal Burgers vector. (c) Local density of states (LDOS) of the hex-Ge supercell modeled in panels a and $\mathrm{b}$ averaged in the $\langle 0001\rangle$ direction, which is the plane perpendicular to the $\langle 1010\rangle$ crystallographic direction. The supercell that contains the $I_{3}$ $\mathrm{BSF}$ and the local band structure plotted along the $\langle 10 \overline{10}\rangle$ clearly shows no midgap states.
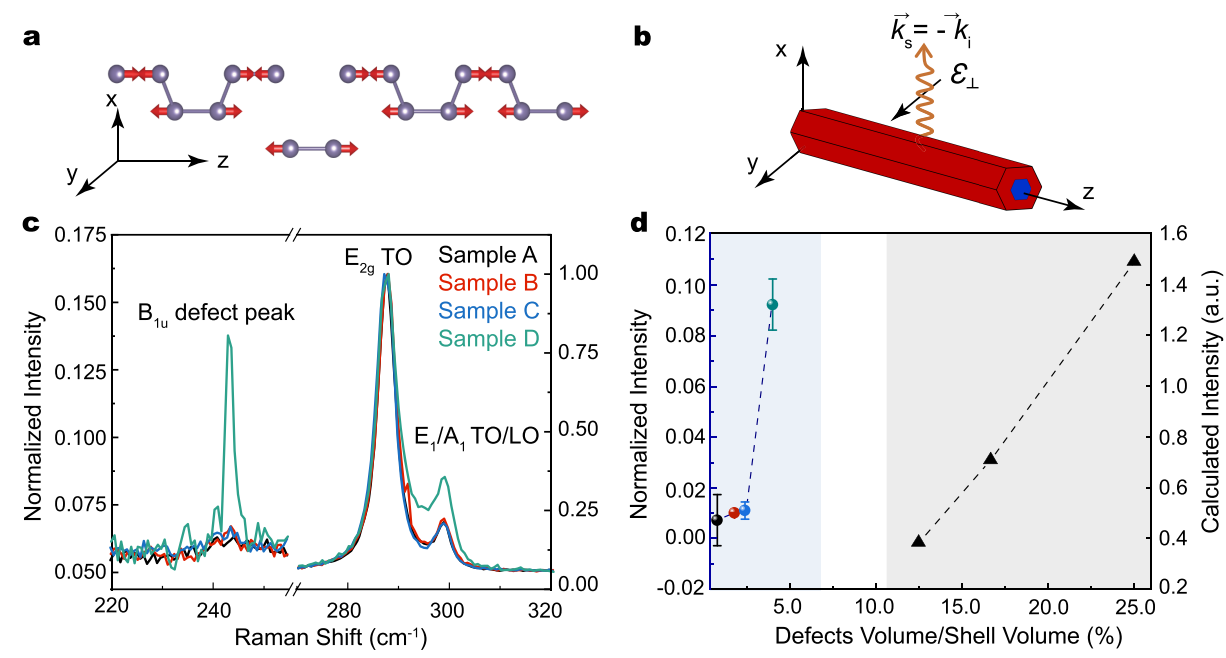

Figure 4. Raman fingerprint of the $I_{3}$ BSF. (a) Calculated atomic displacement vectors of the defect-enabled phonon mode at $241 \mathrm{~cm}^{-1}$ at the $\Gamma$ point for the smallest cell. (b) Scheme of the scattering configurations employed in the Raman measurements. (c) Raman spectra of four hex-Ge nanowires that differ in their $I_{3}$ BSF defect densities. The spectra have been normalized to the intensity of the $E_{2 g}$ mode for a direct comparison of the defect peak intensities. For each sample, several nanowires (two to six) were measured, and the average results are shown in panel d. (d) Left scale, circular symbols: intensity of the $\sim 244 \mathrm{~cm}^{-1}$ peak relative to $E_{2 \mathrm{~g}}$ intensity as a function of defected shell volume percent. Right scale, triangular symbols: theoretical values. Notice that the scales of the $y$-axes of the experimental and theoretical intensities are different, as they are not directly comparable. Experimental error bars on the $y$-scale represent the half-difference between the maximum and minimum value found for that sample, and the error bars on the $x$-scale come from the standard deviation of the estimated defect density. The lines are guides to the eyes.

Supporting Figure 9. Importantly, this means that the electronic properties of a hex-Ge crystal are not affected by the $I_{3} \mathrm{BSF}$ and their terminating dislocations, which is the dominant defect in our hexagonal group IV nanocrystals.

We have analyzed the photoluminescence efficiency of hexGe and hex-SiGe nanowires containing $I_{3}$ BSFs elsewhere, ${ }^{1}$ and we concluded that we have emission from band-to-band recombination. We have not observed a substantial difference in the photoluminescence intensity or spectral line shape between nanowires with different defect densities. The photoluminescence quenching rates (the decrease of the PL intensity with increasing temperature) in defected hex-Ge and hex- $\mathrm{Si}_{0.20} \mathrm{Ge}_{0.80}$ nanowires are very comparable to those observed in high-quality III/V semiconductor nanowires. Additional data show that our hex- $\mathrm{Si}_{0.20} \mathrm{Ge}_{0.80}$ nanowires were capable of reaching the radiative limit up to room 
temperature, indicating a low density of nonradiative centers. The slightly lower photoluminescence quenching rate in hexGe compared to that in hex-SiGe indicates that this conclusion should also be valid for hex-Ge. These observations conclusively show that the $I_{3}$ defects do not significantly degrade the photoluminescence. Although we cannot exclude the idea that $I_{3}$ defects provide a shallow defect level with a low photoluminescence quenching rate, our experimental results support the theoretical claim that the $I_{3}$ BSF and its terminating dislocations do not provide a strong photoluminescence quenching center within the bandgap.

A complementary method for the structural characterization of the $I_{3}$ defects is Raman spectroscopy. We have computed the Raman spectra of $I_{3}$-defected hex-Ge supercells of different sizes by employing density functional perturbation theory (DFPT); for details, see the Methods section of the Supporting Information. In addition to the expected $E_{2 g}$ and $\mathrm{TO} / \mathrm{LO}$ peaks, ${ }^{21,22}$ the computed spectra of defected hex-Ge exhibit a phonon mode at $241 \mathrm{~cm}^{-1}$, which is absent in pure hex-Ge and does not result from the artificial folding imposed by the periodic boundary conditions (for more details, see Supporting Figure 12). The atomic displacements of the 241 $\mathrm{cm}^{-1}$ mode at the $\Gamma$-point were also calculated and are shown in Figure 4a. The motion is not localized at the stacking faults, but it involves all atoms in the unit cell and is oriented along the $z$-axis. Raman scattering experiments were performed on individual hex-GaAs/Ge core/shell nanowires with varying defect densities. The measured nanowires were also examined by TEM, and the defect densities were calculated in terms of the percentage of defected volume over the total shell volume; for details see Supporting Figures 10 and 11 and Supporting Information S3. Figure $4 \mathrm{~b}$ exemplifies the backscattering geometry adopted in the Raman measurements and calculations and the polarization of the incoming and collected photons. In Figure 4c, we compare the Raman spectra of several GaAs/Ge core/shell nanowires, which differ in the density of the $I_{3}$ BSFs in the hexagonal crystal. The spectra of all the nanowires display the transverse optical $E_{2 \mathrm{~g}}$ mode and the degenerate transverse and longitudinal optical $A_{1 \mathrm{~g}}$ and $E_{1 \mathrm{~g}}$ modes, respectively, which are typical of hex-Ge. ${ }^{21,22}$ In addition to this, we observe a weak feature at $244 \mathrm{~cm}^{-1}$ with a varying intensity among the nanowire samples. To directly compare the spectral weight of this peak between different samples, we normalized each spectrum to the intensity of the $E_{2 g}$ mode. We fitted the spectra with Lorentzian curves to extract the intensities of the $244 \mathrm{~cm}^{-1}$ modes relative to those of the $E_{2 g}$ modes. In Figure $4 \mathrm{~d}$, we summarize the relative intensities as a function of the percentage of the defected nanowire shell volume. We observe a direct proportionality between the intensity and the defect density for defect densities above $1.5 \%$ of the shell volume; below this threshold, the experimental intensities are very low and similar. Considering the non-localized nature of the vibration and the fact that the phonon dispersion of bulk hex-Ge has a mode at that frequency with no Raman activity, we attribute the observation of the $244 \mathrm{~cm}^{-1}$ peak to the presence of the $I_{3}$ BSF that alters the Raman selection rules, thus making a silent mode detectable. We suggest that the observation of a threshold $I_{3}$ BSF density is due to the fact that these defects might be too diluted below a certain density to significantly enhance the intensity of the $244 \mathrm{~cm}^{-1}$ phonon mode. Overall, these results indicate that Raman spectroscopy is a powerful non-destructive tool for assessing the density of $I_{3}$ BSFs in group IV semiconductor nanostructures.

In summary, we have identified and characterized the atomistic microstructure of a unique planar intrinsic basal defect, which is dominant in the hexagonal group IV family of materials. The investigation of the planar extension of such a defect revealed that it is formed by an $I_{3}$ BSF, and its atomic stacking exhibited a triangular shape terminated by two partial dislocation dipoles. The $I_{3}$ BSF is the dominant stacking fault embedded in the hexagonal crystal matrix. In addition to TEM, we confirmed that $I_{3}$ BSFs can also be identified by Raman spectroscopy by observing a distinct phonon mode enabled by the stacking fault, which is absent in the pure-phase hexagonal crystal. Eventually, the electronic band structure calculations showed that the $I_{3}$ BSF and its terminating dislocations do not introduce states in the energy gap of hex-Ge and hex-Si. These theoretical calculations are consistent with the reported efficient light emission from hex-Ge and hex-SiGe alloys with $I_{3}$ BSFs. The identification of the dominant defects in a newly discovered class of materials such as the hexagonal group IV family is essential for the development of well-established technology for future optoelectronic devices.

\section{ASSOCIATED CONTENT}

\section{SI Supporting Information}

The Supporting Information is available free of charge at https://pubs.acs.org/doi/10.1021/acs.nanolett.1c00683.

Materials synthesis, TEM characterization, molecular dynamics simulations, stacking fault-energy calculations, electronic band structure calculations, experimental Raman spectroscopy measurements, theoretical Raman calculations, $I_{3}$ BSF visualizations and comparison with the literature, and quantification and volume fraction calculations of the defects (PDF)

Atomic models of the simulated hex-Ge and hex-Si supercells containing the $I_{3}$ defect (ZIP)

\section{AUTHOR INFORMATION}

\section{Corresponding Author}

Marcel A. Verheijen - Department of Applied Physics, Eindhoven University of Technology, $5600 \mathrm{MB}$ Eindhoven, The Netherlands; Eurofins Materials Science Netherlands BV, 5656 AE Eindhoven, The Netherlands; (1) orcid.org/00000002-8749-7755; Email: m.a.verheijen@tue.nl

\section{Authors}

Elham M. T. Fadaly - Department of Applied Physics, Eindhoven University of Technology, $5600 \mathrm{MB}$ Eindhoven, The Netherlands; o orcid.org/0000-0001-7074-8784

Anna Marzegalli - Dipartimento di Fisica, Politecnico di Milano, 22100 Como, Italy; L-NESS and Dipartimento di Scienza dei Materiali, Università di Milano-Bicocca, 20125 Milano, Italy

Yizhen Ren - Department of Applied Physics, Eindhoven University of Technology, $5600 \mathrm{MB}$ Eindhoven, The Netherlands

Lin Sun - Institut für Festkörpertheorie und -optik, FriedrichSchiller-Universität Jena, 07743 Jena, Germany

Alain Dijkstra - Department of Applied Physics, Eindhoven University of Technology, 5600 MB Eindhoven, The Netherlands; 이이.org/0000-0003-2661-5460 
Diego de Matteis - Departement Physik, Universität Basel, 4056 Basel, Switzerland

Emilio Scalise - L-NESS and Dipartimento di Scienza dei Materiali, Università di Milano-Bicocca, 20125 Milano, Italy; ○orcid.org/0000-0003-1465-0939

Andrey Sarikov - L-NESS and Dipartimento di Scienza dei Materiali, Università di Milano-Bicocca, 20125 Milano, Italy; V. Lashkarev Institute of Semiconductor Physics, National Academy of Sciences of Ukraine, 03028 Kyiv, Ukraine

Marta De Luca - Departement Physik, Universität Basel, 4056 Basel, Switzerland; 이이.org/0000-0001-60613261

Riccardo Rurali - Institut de Ciencia de Materials de Barcelona, ICMAB-CSIC, 08193 Bellaterra, Spain; (1) orcid.org/0000-0002-4086-4191

Ilaria Zardo - Departement Physik, Universität Basel, 4056 Basel, Switzerland; 이이.orid.o000-0002-8685-2305

Jos E. M. Haverkort - Department of Applied Physics, Eindhoven University of Technology, $5600 \mathrm{MB}$ Eindhoven, The Netherlands; (1) orcid.org/0000-0003-3051-673X

Silvana Botti - Institut für Festkörpertheorie und -optik, Friedrich-Schiller-Universität Jena, 07743 Jena, Germany; (1) orcid.org/0000-0002-4920-2370

Leo Miglio - L-NESS and Dipartimento di Scienza dei Materiali, Università di Milano-Bicocca, 20125 Milano, Italy; ○ orcid.org/0000-0002-1329-527X

Erik P. A. M. Bakkers - Department of Applied Physics, Eindhoven University of Technology, 5600 MB Eindhoven, The Netherlands

Complete contact information is available at:

https://pubs.acs.org/10.1021/acs.nanolett.1c00683

\section{Author Contributions}

$\triangle$ These authors contributed equally to this work. E.M.T.F. and M.A.V. conceived the idea. E.M.T.F. grew the GaAs/Ge nanowires and Y.R. grew the $\mathrm{GaP} / \mathrm{Si}$ nanowires. M.A.V. performed the TEM characterization of the nanowires. A.M., E.S., and A.S.,L.M. performed the atomistic modeling and molecular dynamics simulations. D.D.M. and M.D.L. performed the Raman measurements. D.D.M., M.D.L., and I.Z. analyzed and interpreted the Raman experimental results. R.R. performed the Raman theoretical calculations. L.S. and S.B. performed the band structure calculations. A.D. and J.E.M.H. performed the optical characterization. I.Z., S.B., L.M., E.P.A.M.B., and M.A.V. supervised the project. The manuscript was written by E.M.T.F. through the contributions of all authors. All authors have given approval to the final version of the manuscript.

\section{Funding}

This project has received funding from the European Union's Horizon 2020 research and innovation program under Grant agreement no. 735008 (SiLAS). I.Z. acknowledges financial support from the European Research Council (ERC) under the European Union's Horizon 2020 research and innovation program (Grant agreement no. 756365). M.D.L. acknowledges support from the Swiss National Science Foundation Ambizione grant (Grant no. PZ00P2_179801). R.R. acknowledges financial support by the Ministerio de Economia, Industria y Competitividad (MINECO) under Grant FEDER-MAT2017-90024-P, by the Severo Ochoa Centres of Excellence Program under Grant SEV-2015-049,6 and by the Generalitat de Catalunya under Grant no. 2017 SGR 1506. L.S. acknowledges financial support from the China Scholarship Council. S.B. acknowledges funding from the Volkswagen Stiftung (Momentum) through the project "Dandelion" and the DFG through projects SFB-1375 and BO4280/8-1. Computational resources were also provided by the Leibniz Supercomputing Center through projects pr48je and pr62ja.

\section{Notes}

The authors declare no competing financial interest.

\section{ACKNOWLEDGMENTS}

We thank Dr. Sebastian Kölling for performing the focused ion beam experiment to prepare a lamella of the Hex-GaAs/Ge core/shell nanowire structure for TEM characterization. Solliance and the Dutch province of Noord Brabant are acknowledged for funding the TEM facility. E.S. and A.S. acknowledge the CINECA consortium for the availability of high-performance computing resources, which were granted under the ISCRA initiative.

\section{REFERENCES}

(1) Fadaly, E. M. T.; Dijkstra, A.; Suckert, J. R.; Ziss, D.; van Tilburg, M. A. J.; Mao, C.; Ren, Y.; van Lange, V. T.; Korzun, K.; Kölling, S.; et al. Direct-Bandgap Emission from Hexagonal $\mathrm{Ge}$ and $\mathrm{SiGe}$ Alloys. Nature 2020, 580 (7802), 205-209.

(2) Barth, S.; Seifner, M. S.; Maldonado, S. Metastable Group IV Allotropes and Solid Solutions: Nanoparticles and Nanowires. Chem. Mater. 2020, 32 (7), 2703-2741.

(3) Dushaq, G.; Nayfeh, A.; Rasras, M. Hexagonal Germanium Formation at Room Temperature Using Controlled Penetration Depth Nano-Indentation. Sci. Rep. 2019, 9 (1), 1593.

(4) Qiu, Y.; Bender, H.; Richard, O.; Kim, M. S.; Van Besien, E.; Vos, I.; De Potter De Ten Broeck, M.; Mocuta, D.; Vandervorst, W. Epitaxial Diamond-Hexagonal Silicon Nano-Ribbon Growth on (001) Silicon. Sci. Rep. 2015, 5 (1), 12692.

(5) Vincent, L.; Patriarche, G.; Hallais, G.; Renard, C.; Gardès, C.; Troadec, D.; Bouchier, D. Novel Heterostructured Ge Nanowires Based on Polytype Transformation. Nano Lett. 2014, 14 (8), 48284836.

(6) Arbiol, J.; Fontcuberta I Morral, A.; Estradé, S.; Peiró, F.; Kalache, B.; Roca I Cabarrocas, P.; Morante, J. R. Influence of the (111) Twinning on the Formation of Diamond Cubic/Diamond Hexagonal Heterostructures in Cu-Catalyzed Si Nanowires. J. Appl. Phys. 2008, 104 (6), 064312.

(7) Zhang, Y.; Iqbal, Z.; Vijayalakshmi, S.; Qadri, S.; Grebel, H. Formation of Hexagonal-Wurtzite Germanium by Pulsed Laser Ablation. Solid State Commun. 2000, 115 (12), 657-660.

(8) Xiao, S. Q.; Pirouz, P. On Diamond-Hexagonal Germanium. J. Mater. Res. 1992, 7 (6), 1406-1412.

(9) Hauge, H. I. T.; Conesa-Boj, S.; Verheijen, M. A.; Koelling, S.; Bakkers, E. P. A. M. Single-Crystalline Hexagonal Silicon-Germanium. Nano Lett. 2017, 17 (1), 85-90.

(10) Hauge, H. I. T.; Verheijen, M. A.; Conesa-Boj, S.; Etzelstorfer, T.; Watzinger, M.; Kriegner, D.; Zardo, I.; Fasolato, C.; Capitani, F.; Postorino, P.; et al. Hexagonal Silicon Realized. Nano Lett. 2015, 15 (9), 5855-5860.

(11) Algra, R. E.; Hocevar, M.; Verheijen, M. A.; Zardo, I.; Immink, G. G. W.; Van Enckevort, W. J. P.; Abstreiter, G.; Kouwenhoven, L. P.; Vlieg, E.; Bakkers, E. P. A. M. Crystal Structure Transfer in Core/ Shell Nanowires. Nano Lett. 2011, 11 (4), 1690-1694.

(12) Johansson, J.; Bolinsson, J.; Ek, M.; Caroff, P.; Dick, K. A. Combinatorial Approaches to Understanding Polytypism in III-V Nanowires. ACS Nano 2012, 6 (7), 6142-6149.

(13) Zakharov, D. N.; Liliental-Weber, Z.; Wagner, B.; Reitmeier, Z. J.; Preble, E. A.; Davis, R. F. Structural TEM Study of Nonpolar APlane Gallium Nitride Grown on (11 $\overline{2} 0) 4 \mathrm{H}-\mathrm{SiC}$ by Organometallic 
Vapor Phase Epitaxy. Phys. Rev. B: Condens. Matter Mater. Phys. 2005, 71 (23), 235334.

(14) Stampfl, C.; Van De Walle, C. G. Energetics and Electronic Structure of Stacking Faults in AlN, GaN, and InN. Phys. Rev. B: Condens. Matter Mater. Phys. 1998, 57 (24), R15052.

(15) Xin, H. L.; Muller, D. A. Three-Dimensional Imaging in Aberration-Corrected Electron Microscopes. Microsc. Microanal. 2010, 16, 445-455.

(16) Conesa-Boj, S.; Hauge, H. I. T.; Verheijen, M. A.; Assali, S.; Li, A.; Bakkers, E. P. A. M.; Fontcuberta I Morral, A. Cracking the Si Shell Growth in Hexagonal GaP-Si Core-Shell Nanowires. Nano Lett. 2015, 15 (5), 2974-2979.

(17) Sanchez, A. M.; Gott, J. A.; Fonseka, H. A.; Zhang, Y.; Liu, H.; Beanland, R. Stable Defects in Semiconductor Nanowires. Nano Lett. 2018, 18 (5), 3081-3087.

(18) Hirth, J. P.; Lothe, J. Theory of Dislocations, 2nd ed.; Wiley: New York, NY, 1982.

(19) Tersoff, J. Modeling Solid-State Chemistry: Interatomic Potentials for Multicomponent Systems. Phys. Rev. B: Condens. Matter Mater. Phys. 1989, 39 (8), 5566-5568.

(20) Rauch, T.; Marques, M. A. L.; Botti, S. Local Modified BeckeJohnson Exchange-Correlation Potential for Interfaces, Surfaces, and Two-Dimensional Materials. J. Chem. Theory Comput. 2020, 16 (4), 2654-2660.

(21) Fasolato, C.; De Luca, M.; Djomani, D.; Vincent, L.; Renard, C.; Di Iorio, G.; Paillard, V.; Amato, M.; Rurali, R.; Zardo, I. Crystalline, Phononic, and Electronic Properties of Heterostructured Polytypic Ge Nanowires by Raman Spectroscopy. Nano Lett. 2018, 18 (11), 7075-7084.

(22) De Matteis, D.; De Luca, M.; Fadaly, E. M. T.; Verheijen, M. A.; López-Suárez, M.; Rurali, R.; Bakkers, E. P. A. M.; Zardo, I. Probing Lattice Dynamics and Electronic Resonances in Hexagonal Ge and $\mathrm{Si}_{x} \mathrm{Ge}_{1-x}$ Alloys in Nanowires by Raman Spectroscopy. ACS Nano 2020, 14 (6), 6845-6856. 